\title{
Drosophila Fos mediates ERK and JNK signals via distinct phosphorylation sites
}

\author{
Laura Ciapponi, ${ }^{1,2}$ David B. Jackson, ${ }^{1,4}$ Marek Mlodzik, ${ }^{1,3}$ and Dirk Bohmann ${ }^{1,2,5}$ \\ ${ }^{1}$ European Molecular Biology Laboratory, D-69117 Heidelberg, Germany; ${ }^{2}$ Center for Cancer Biology, Aab Institute of \\ Biomedical Sciences, University of Rochester School of Medicine and Dentistry, Rochester, New York 14642, USA; ${ }^{3}$ Mount \\ Sinai School of Medicine, Department of Cell Biology and Anatomy, New York, New York 10029, USA
}

\begin{abstract}
During Drosophila development Fos acts downstream from the JNK pathway. Here we show that it can also mediate ERK signaling in wing vein formation and photoreceptor differentiation. Drosophila JNK and ERK phosphorylate D-Fos with overlapping, but distinct, patterns. Analysis of flies expressing phosphorylation site point mutants of D-Fos revealed that the transcription factor responds differentially to JNK and ERK signals. Mutations in the phosphorylation sites for JNK interfere specifically with the biological effects of JNK activation, whereas mutations in ERK phosphorylation sites affect responses to the EGF receptor-Ras-ERK pathway. These results indicate that the distinction between ERK and JNK signals can be made at the level of D-Fos, and that different pathway-specific phosphorylated forms of the protein can elicit different responses.
\end{abstract}

[Key Words: Fos; JNK; ERK; Drosophila; signal specificity]

Received February 5, 2001; revised version accepted April 17, 2001

The activation of a given signaling pathway can have a range of biological effects depending on the cellular context. Distinct cellular responses of this kind may be determined by the particular combination of responsive transcription factors expressed in the signal-receiving cell. The presence of a certain set of transcription factors can thus predetermine the specific genetic response of a cell to a pleiotropically acting signal (Simon 2000).

This simple concept becomes complicated if transcription factors serve as nodes targeted by more than one signal transduction pathway, as has been described in a number of cases based on tissue culture experiments. Well-known examples include the Ets family factor Elk (Treisman 1996) or the leucine zipper factor CREB (De Cesare et al. 1999), both of which serve as convergence points for several signaling pathways. Mechanistically this is explained by the ability of multiple, differently regulated kinases to phosphorylate such "multifunctional" transcription factors and thus to modulate their ability to activate target genes.

Here we investigated how the Jun-N-terminal kinase (JNK) and the extracellular regulated kinase (ERK) signal transduction pathways, two related but distinct MAP kinase signaling cassettes, converge on a single transcrip-

\footnotetext{
${ }^{4}$ Present address: LION Bioscience AG, 69120 Heidelberg, Germany. ${ }^{5}$ Corresponding author.

E-MAIL Dirk_Bohmann@urmc.rochester.edu; FAX (716) 273-1450. Article and publication are at www.genesdev.org/cgi/doi/10.1101/ $\operatorname{gad} .886301$.
}

tion factor, D-Fos, during the development of Drosophila.

Fos and its partner molecule Jun are members of the AP-1 family of bZIP transcription factors (Leppä and Bohmann 1999; Wisdom 1999). Fos and Jun are paradigmatic examples for pleiotropically acting gene regulators, both implicated in the nuclear response to a wide variety of extracellular signals. Jun has been studied most extensively in this regard. It is the name-giving substrate of the MAP kinase JNK (Hibi et al. 1993; Dérijard et al. 1994). In vertebrate cells, JNK signaling via Jun mediates the cellular response to stress and apoptotic signals. In addition, studies in fibroblasts, PC12 cells, and Drosophila have indicated that c-Jun can also relay the cellular response to ERK activation (Pulverer et al. 1991; Peverali et al. 1996; Kockel et al. 1997; Leppä et al. 1998).

Fos is involved in the cellular response to ERK and JNK as well; however, the mechanistic understanding of the role of Fos as MAPK effector is less advanced than the one of Jun. c-Fos, the vertebrate homolog of D-Fos, becomes hyperphosphorylated in response to a range of stimuli. Although the functional consequences of this phosphorylation have not been investigated in great detail, it correlates with the activation of c-Fos target genes, especially in response to serum stimulation. Several MAP kinases or MAP kinase-regulated protein kinases have been shown to phosphorylate c-Fos in vitro. These include ERK, RSK1, and FRK (Deng and Karin 1994; Chen et al. 1996).

Drosophila has proven to be a very well-suited experi- 
mental system for the analysis of MAPK signaling. The fruit fly permits biochemical as well as genetic approaches toward elucidation of molecular mechanisms and biological functions of signaling pathways such as the ERK and JNK cascades. The only known Drosophila ERK is Rolled (Biggs et al. 1994). It acts downstream from at least five receptor tyrosine kinases (RTKs; Gabay et al. 1997). For the purpose of this study, we consider the Drosophila homolog of the epidermal growth factor receptor (DER) and the eye-specific RTK Sevenless (Sev). DER and Sev activate a similar signal transduction pathway, which involves Ras, Raf, and Rolled, but differ in their biological functions. DER is probably the most pleiotropic of the Drosophila RTKs (Schweitzer and Shilo 1997; Freeman 1998). It participates in a large number of developmental decisions. For example, it controls cell proliferation and patterning in imaginal discs and thus determines the formation of many adult structures such as the wing, eye, and leg. In the eye imaginal disc, DER and Sev act in concert to control, among others events, the differentiation of photoreceptor neurons (Rcells; Simon 1994; Wassarman et al. 1995). Previous studies have indicated that RTK-dependent R-cell development can be mediated by D-Jun (Bohmann et al. 1994; Treier et al. 1995; Peverali et al. 1996), even though lossof-function experiments show that the protein is not essential for the process (Hou et al. 1997; Kockel et al. 1997). A dominant-negative form of rat c-Fos, when expressed in the developing eye, interferes with Sev-dependent $\mathrm{R}$-cell recruitment, indicating a role for Fos in Rcell differentiation as well (Bohmann et al. 1994). The precise relationship between Fos, Jun, and the RTK-dependent activation of ERK in Drosophila eye and wing development, however, is unclear.

During the development of the gut, D-Fos appears to mediate the regulation of the homeotic gene labial, a key determinant of intestinal morphogenesis (Szüts and Bienz 2000b). In this system, D-Fos is regulated by DER, but it is not clear yet whether the RTK signal controls D-Fos expression, phosphorylation, or both (Eresh et al. 1997; Riese et al. 1997; Szüts et al. 1998).

The role of D-Fos as a mediator of JNK signals is better defined than its function as an ERK effector, however. Like the ERK pathway, the JNK pathway controls several events during Drosophila development (Kockel et al. 2001). Dorsal closure is the best described example. In this process, the epidermis of the developing embryo moves dorsalward to close a region that until then is covered only by a transient embryonic cell layer, called amnioserosa (Knust 1996). Mutations affecting components of the JNK pathway, such as the D-JNKK Hemipterous (Hep) and the D-JNK Basket (Bsk), as well as D-Jun and D-Fos, result in defective dorsal closure (Noselli 1998; Stronach and Perrimon 1999). Genetic interaction studies indicated that D-Fos and D-Jun are both required downstream from Bsk for dorsal closure to proceed (Riesgo-Escovar et al. 1996; Hou et al. 1997; Kockel et al. 1997; Riesgo-Escovar and Hafen 1997a; Sluss and Davis 1997; Zeitlinger et al. 1997). D-Jun serves as a direct target for Bsk in vitro and a constitutively active, pseu- dophosphorylated form of c-Jun can partially rescue bsk mutants, indicating that D-Jun phosphorylation by Bsk is essential for dorsal closure (Riesgo-Escovar et al. 1996; Sluss et al. 1996; Hou et al. 1997). An additional JNKregulated process in Drosophila that is phenomenologically similar to dorsal closure is thorax closure, the joining of the collateral wing imaginal disc at the dorsal midline during pupariation. Fos is required to mediate the JNK signal in the control of thorax closure (Agnes et al. 1999; Zeitlinger and Bohmann 1999; Martin-Blanco et al. 2000).

Here we present evidence that D-Fos presents a node for the intersection of ERK and JNK signal transduction pathways during a number of developmental situations, and we suggest a mechanism that mediates the specificity of signal responses.

\section{Results}

\section{D-Fos directs wing vein formation}

In addition to its embryonic expression pattern, D-Fos can be detected at later stages of development, for example, in wing and eye imaginal disc (Zeitlinger and Bohmann 1999; J. Zeitlinger, unpubl.). To gain insight into late developmental functions of D-Fos, we began to analyze its contribution to wing development.

The early lethality of Drosophila fos mutants ruled out a direct assessment of imaginal disc development in flies lacking $D$-fos. Generation of somatic mutant clones of cells deficient for $D$-fos was not feasible because the only extant alleles, $\mathrm{kay}^{1}$ and $\mathrm{kay}^{2}$, are not suitable for such experiments. kay $^{2}$ represents a hypomorphic mutation of unknown nature. Therefore, the occasional viable homozygous flies (escapers) that are recovered from this stock might not display the full range of phenotypes that can arise from defects in D-fos. The null allele $\mathrm{kay}^{1}$, on the other hand, causes cell lethality in imaginal disc cell clones homozygous for this mutation. It is not clear, however, whether this drastic phenotype is caused by a loss of D-Fos function, as it cannot be rescued by transgenic D-Fos expression (J. Zeitlinger, unpubl.). This may be due to the fact that the $k a y^{1}$ mutation consists of a deletion that removes more than one lethal complementation group (J. Zeitlinger, U. Gritzan, H. Jasper, L. Ciapponi, unpubl.). Therefore, we opted for an alternative approach and examined fly strains expressing a dominantnegative mutant form of D-Fos (subsequently referred to as D-Fos ${ }^{\text {bZIP }}$. This truncated version of D-Fos consists of the isolated bZIP domain. Thus, it can dimerize with endogenous Fos partner proteins such as D-Jun and bind to DNA, but it lacks the ability to stimulate transcription (Eresh et al. 1997).

A number of observations have shown that the expression of D-Fos ${ }^{\text {bZIP }}$ causes phenotypes specifically resembling those elicited by $D$-fos loss-of-function alleles $\left(\right.$ kay $^{1}$ and kay $^{2}$ ): (1) When expressed in epidermal cells during embryogenesis, D-Fos ${ }^{\text {bZIP }}$ causes a dorsal anterior hole, which phenocopies the dorsal closure defect of fosdeficient embryos (Zeitlinger et al. 1997); (2) expression 
of D-Fos ${ }^{\text {bZIP }}$ in the dorsal part of the wing imaginal disc epithelium, from which the adult thoracic body wall derives, results in a cleft along the dorsal midline, as do $D$-fos mutant alleles (Zeitlinger and Bohmann 1999); (3) expression of D-Fos ${ }^{\text {bZIP }}$ in the embryonic endoderm elicits a midgut phenotype and reduces labial expression, reminiscent of kay mutants (Eresh et al. 1997; Szüts and Bienz 2000b). D-Fos ${ }^{\text {bZIP }}$, therefore, is a suitable tool with which to analyze the function and the regulation of DFos in later developmental processes such as imaginal disc morphogenesis.

Using the UAS/Gal4 system, D-Fos ${ }^{\text {bZIP }}$ was expressed in regions of the wing imaginal disc that give rise to the entire adult wing structure or parts of it (Fig. 1, blue areas). Expression under the control of the three Gal4 drivers used, dpp Gal4, en Gal4, and sd Gal4, resulted in loss of vein material in the respective expression domains. The expression of D-Fos ${ }^{\text {bZIP }}$ under $d p p$ control, between the longitudinal veins II and III, causes disappearance of the anterior crossvein (Fig. 1A, arrow). When driven in the posterior compartment by en Gal4, DFos ${ }^{\text {bZIP }}$ causes partial loss of the longitudinal vein V (Fig.

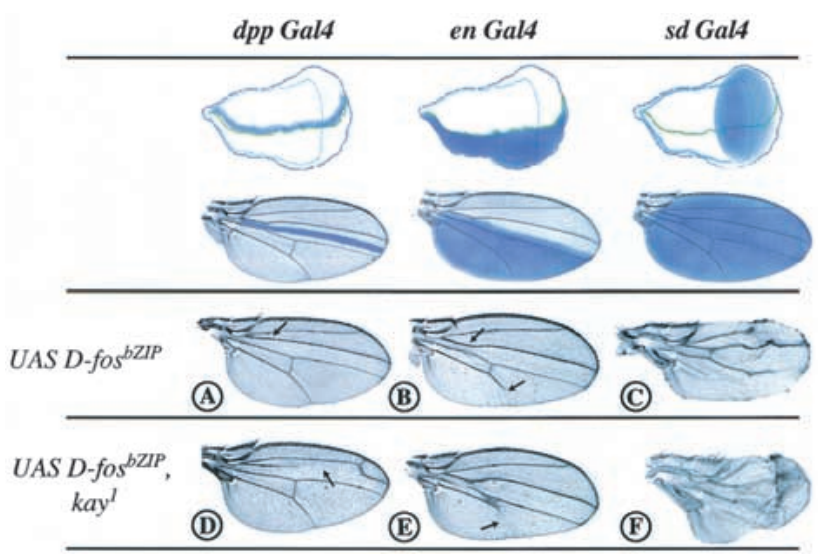

Figure 1. Expression of a dominant-negative mutant form of D-Fos in the wing causes loss of vein material. Adult wings of flies expressing a dominant-negative form of D-Fos (D-Fos ${ }^{\text {bZIP }}$ ) under the control of $d p p$, engrailed, or scalloped GAL4 drivers (dpp GAL4, en GAL4, and sd GAL4, respectively) in a wild-type (UAS D-fos ${ }^{b Z I P}$ ), or kayak (UAS D-fos ${ }^{\text {bZIP }}$, kay $^{1}$ ) background. The blue areas demarcate the expression pattern of each driver in the imaginal disc (top) and the adult wing (bottom). Note that the expression of D-Fos ${ }^{\mathrm{bZIP}}$, using either driver, causes loss of vein material and that this effect is enhanced in the fos mutant background $\left(\right.$ kay $\left.^{1}\right)$. (A) dpp GAL4/+; UAS D-fos ${ }^{b Z I P} /+$ causes loss of the anterior crossvein (black arrow); (B) en GAL4/+; UAS $D-f O s^{b Z I P} /+$ causes loss of the anterior crossvein and partial loss of the longitudinal vein $\mathrm{V}$ (black arrows); $(C)$ sd GAL4/+; UAS $D-f O S^{b Z I P} /+$, note that the longitudinal vein $\mathrm{V}$ and part of veins II and IV are missing. (D) D-Fos ${ }^{\text {bZIP }}$ expressed in the $d p p$ compartment of a kay ${ }^{1}$ heterozygote causes almost complete loss of the longitudinal vein III (black arrow; due to weak $d p p$ expression in the entire anterior compartment). (E) en GAL4 driving D-Fos ${ }^{\text {bZIP }}$ expression in $\mathrm{kay}^{1} /+$ causes the disappearance of longitudinal vein $\mathrm{V}$ (black arrow) more completely as compared with $B$. $(F)$ In $s d$ GAL4/+; UAS fos ${ }^{b Z I P}, \mathrm{kay}^{1} /+$ note that the all wing vein material is strongly reduced.
$1 \mathrm{~B}$, bottom arrow). Finally, if the expression of D-Fos ${ }^{\text {bzIP }}$ is driven by $s d$ Gal4 in the entire wing pouch, the phenotype is most striking, displaying a partial loss of veins II and IV and of almost the entire vein V (Fig. 1C). Significantly, the D-Fos ${ }^{\mathrm{bZIP}}$-dependent loss-of-vein phenotype is enhanced in a heterozygous $D$-fos loss-of-function $\left(\mathrm{kay}^{1}\right)$ background, that is, when the endogenous $D$-fos gene dose is reduced. This confirms that the observed effect of D-Fos ${ }^{\text {bZIP }}$ is specific and caused by interference with endogenous D-Fos (Fig. 1, cf. A and D, B and $\mathrm{E}$, and $\mathrm{C}$ and $\mathrm{F}$ ).

\section{$D$-Fos as a mediator of the DER pathway in the wing}

The loss of wing vein tissue on expression of D-Fos ${ }^{\text {bZIP }}$ resembles phenotypes resulting from defects in the Drosophila epidermal growth factor receptor (DER) pathway, caused, for example, by loss-of-function alleles of DER itself (Clifford and Schüpbach 1989) or of other genes required for DER signaling, such as rhomboid, vein, ras, and ERK/rolled (Sturtevant et al. 1993; DiazBenjumea and Hafen 1994; Garcia-Bellido et al. 1994). Therefore, the above results might indicate that D-Fos acts as a mediator of the DER/ERK signaling pathway during wing vein differentiation. The artificial activation of this RTK pathway, by gain-of-function alleles or by overexpression of downstream effectors, gives rise to ectopic veins in the wing (Baker and Rubin 1992; Brand and Perrimon 1993; Brunner et al. 1994). To establish whether D-Fos might act epistatically to DER, we examined whether D-Fos ${ }^{\text {bZIP }}$ or a reduced $D$-fos gene dose (kayak alleles) might suppress such a phenotype.

The Ellipse ${ }^{B 1}\left(E l p^{B 1}\right.$; Baker and Rubin 1992) allele of DER represents an activated component of the DER/ERK signaling pathway. In addition to other phenotypes, for example, in the eye (see below), Elp ${ }^{B 1}$ animals consistently develop wings with ectopic wing vein material (Fig. 2A,B, strong and mild phenotypes, respectively). Strikingly, both D-Fos ${ }^{\text {bZIP }}$ expression in the wing imaginal disc (using the 32B Gal4 driver) or the removal of one copy of $D$-fos in a kayheterozygote, suppress this phenotype almost completely (Fig. 2C,D,F; see also the bar graphs for quantification). To confirm that the observed effect is specific and caused by a reduction of endogenous D-Fos function, we performed add-back experiments in which this reduction was compensated by supplying extra wild-type D-Fos from a transgene, driven by the heat shock promoter (hs D-fos; Zeitlinger et al. 1997). Significantly, the presence of the $D$-fos transgene abrogated the suppression of $E l p^{B 1}$ by kay and reinstated the extra vein phenotype caused by elevated DER activity (Fig. 2E and graph). This result confirms that the suppression of the activated DER allele is due to a loss of D-fos activity. Hence, D-Fos mediates wing vein patterning downstream from or in parallel with DER.

\section{Multiple roles of D-Fos in RTK signaling}

Next, we investigated whether D-Fos mediates ERK signaling also during eye morphogenesis (Basler and Hafen 


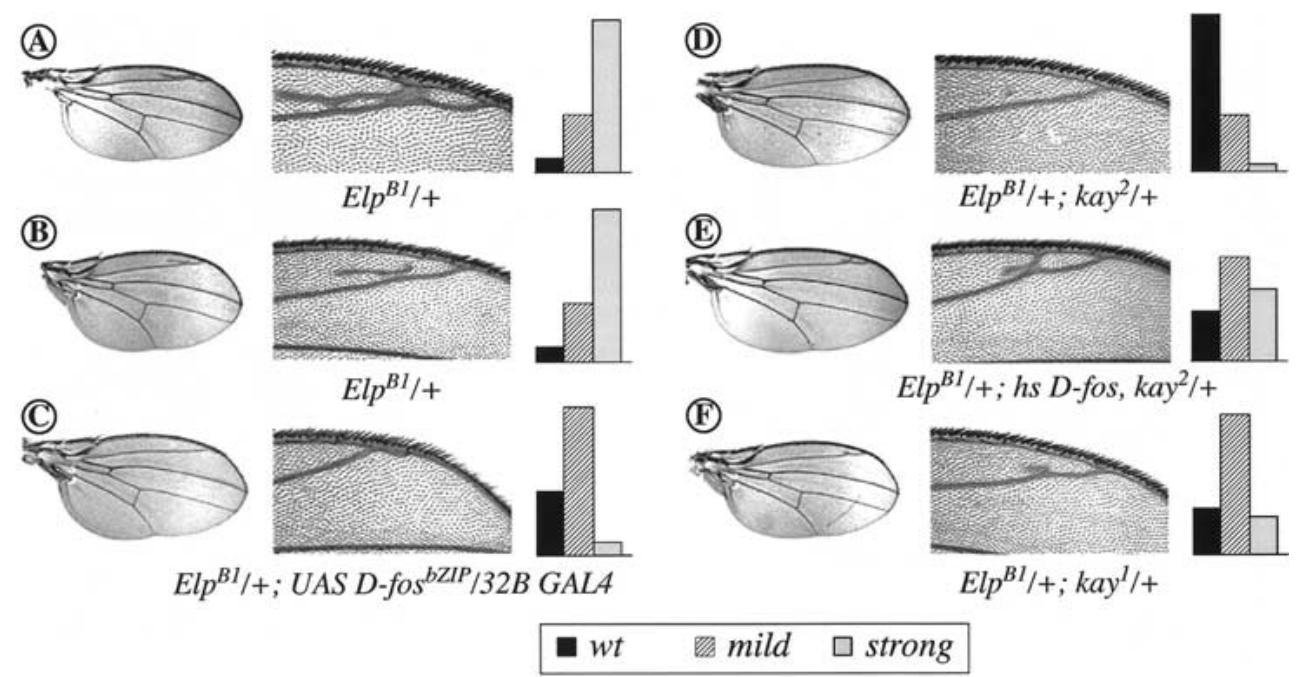

Figure 2. kayak alleles and the dominant-negative mutant form of D-Fos dominantly suppress the phenotype caused by activated DER in the wing. Flies of the $E l p^{B 1} /+$ genotype display a variable extra-vein phenotype ranging from strong $(A)$ to mild $(B)$. The extra vein material (see magnified view next to the wing pictures) is suppressed completely in the presence of $U A S D-f_{O S}{ }^{b Z I P} / 32 B$ GAL4 $(C)$, $k^{k_{y}}{ }^{2}(D)$, or kay ${ }^{1}$ alleles $(F)$. (E) Transgenic expression of full-length D-Fos (hs D-fos) in combination with kay ${ }^{2}$ heterozygosity (or with kay $^{1}$; not shown) in Elp ${ }^{B 1}$ background causes restoration of the extra-vein phenotype (see also graphs). The graphs display the quantified extra-vein phenotype in the different genotypes. Statistical analysis of a percentage of wild-type (black bars), mild (dashed bars), and strong (gray bars) wing phenotypes among the genotypes reported (for each genotype $>200$ flies were analyzed). Note that the restoration of the $E l p^{B 1}$ extra-vein phenotype by $h s D$-fos in a $k a y^{2}$ background is not complete because this result was obtained even without heat shock.

1988; Fortini et al. 1992; Freeman 1996). Defects in photoreceptor differentiation can be induced by the RTK gain-of-function alleles $E l p^{B 1}$ and $s e v^{S 11}$. The $E l p^{B 1}$ allele dominantly causes an abnormal eye phenotype that manifests itself in roughness and the occasional lack of outer photoreceptors (Fig. 3B; Baker and Rubin 1989; Lim et al. 1997). This phenotype can be suppressed largely by the removal of one copy of $D$-fos (Fig. 3C) and restored subsequently by simultaneous transgenic expression of wild-type D-Fos (Fig. 3D). A gain-of-function transgene of the RTK-coding gene sevenless $\left(\mathrm{sev}^{\mathrm{S11}}\right)$ causes the characteristic appearance of ectopic R7 photoreceptor cells in nearly all ommatidia (Fig. 3F; Brunner et al. 1994). The $s e v^{S 11}$ phenotype can be suppressed by the expression of dominant-negative Fos (Bohmann et al. 1994; L. Ciapponi, unpubl.). In flies carrying $\mathrm{sev}^{\mathrm{S11}}$ in a heterozygous kay ${ }^{2}$ background, the ectopic R7 photoreceptor phenotype is suppressed significantly (Fig. 3G); the number of normal ommatidia increases from $5 \%$ to approximately $20 \%$ (Fig. 3I). Reintroduction of $D$-fos by a transgene in this double mutant background restores the percentage of ommatidia with extra photoreceptors observed in $\operatorname{sev}^{S 11}$ heterozygous animals (Fig. $3 \mathrm{H}, \mathrm{I}$ ). Taken together, these results indicate that D-Fos can act as a rate-limiting component downstream from the RTKs Sev and DER during eye development.

\section{$D$-Fos acts as an effector of ERK/Rolled}

Considering that D-Fos is a transcription factor and based on the precedents of D-Jun and c-Fos (Chen et al. 1996; Peverali et al. 1996), the most obvious role for D-
Fos in DER and Sev signal transduction would be that of an effector of the Drosophila MAP kinase Rolled. Therefore, we examined the effect of reducing $D$-fos activity in animals expressing the gain-of-function allele $r 1^{\mathrm{Sem}}$ (Brunner et al. 1994). Expression of $\mathrm{R}^{\mathrm{Sem}}$ under UAS control in the wing imaginal disc resulted in an extravein phenotype, markedly when the flies were reared at $25^{\circ} \mathrm{C}$ and milder at $18^{\circ} \mathrm{C}$ (Fig. 4A,D). Simultaneous expression of D-Fos ${ }^{\text {bZIP }}$ along with $\mathrm{Rl}^{\text {Sem }}$ causes a striking suppression of ectopic vein formation (Fig. 4, cf. A and C; note that for this experiment conditions were chosen in which Fos ${ }^{\text {bZIP }}$ produces only a very mild effect by itself [see legend]), whereas additional expression of full-length D-Fos leads to a strong enhancement of the phenotype (Fig. 4, cf. D and F). To confirm that the observed suppression of the $r l^{\text {Sem }}$ phenotype was not due to an effect of D-Fos on the transgene promoters, we performed a similar genetic interaction experiment using the endogenous $r l^{\text {Sem }}$ gain-of-function allele and $\mathrm{kay}^{2}$ allele. $\mathrm{kay}^{2}$ heterozygosity suppresses the $r l^{\mathrm{Sem}}$-induced extra-vein phenotype (Fig. 4, cf. G and H). This effect is reverted by ubiquitous expression of D-Fos (Fig. 4I), indicating that the suppression is due specifically to the decreased activity of D-Fos. The genetic interactions presented above confirm that the role of D-Fos in RTK signal is that of an effector of Rolled.

\section{D-Fos acts downstream from both ERK and INK signals in the developing eye}

The results described above reveal D-Fos as a downstream component of the ERK signal transduction path- 


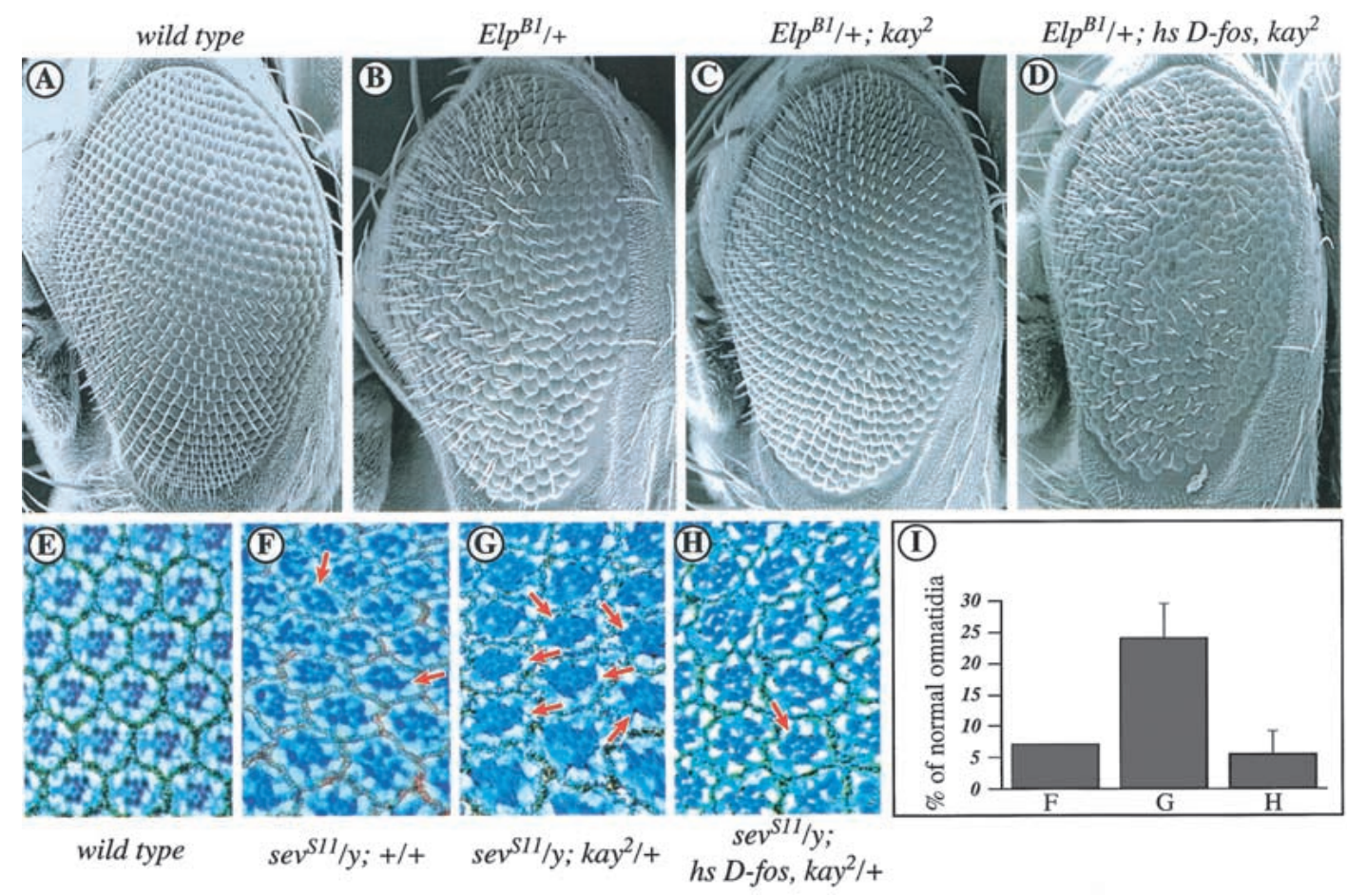

Figure 3. A kayak loss-of-function allele dominantly suppresses the phenotypic effect of activated RTKs in the Drosophila eye. (A-D) Scanning electron micrographs of adult eyes from flies with the indicated genotypes. $(A)$ Wild type. $(B) E l p^{B 1} /+$ causes external roughness. $(C)$ The $E l p^{B 1}$ phenotype can be suppressed by reducing the dosage of $D$-fos to one copy. $(D)$ This suppression can be reverted when exogenous $D$-fos is expressed ubiquitously in the $E l p^{B 1} /+; \mathrm{kay}^{2} /+$ background. $(E-G)$ Tangential eye sections from flies with the indicated genotypes. $(E)$ Wild-type eye section. $(F)$ The sevenless gain-of-function allele, $\mathrm{seV}^{S 11}$, causes a high percentage of ommatidia with supernumerary R7-type photoreceptors. $(G)$ This effect can be suppressed significantly by reduction of $D$-fos activity (normal ommmatidia are indicated by arrows). (H) The suppression is overridden by simultaneous expression of D-Fos. $(I)$ Statistical analysis of the genotypes shown in panels $F-H$; percentage of normal ommatidia \pm S.D. is shown (for each genotype at least three independent eyes and $>300$ ommatidia were analyzed, $P<0.001)$. Note that the expression of exogenous D-Fos in the eye relies on the sevenless enhancer located upstream of the heat shock promoter of the pKB267 P-element vector.

way, yet previous genetic analyses have shown that the transcription factor serves as an effector of JNK (RiesgoEscovar and Hafen 1997a; Zeitlinger et al. 1997). This raises the question of whether the function of D-Fos as recipient of ERK or JNK is mutually exclusive and determined by the cellular context, or whether the transcription factor may mediate both JNK and ERK responses in one tissue or one cell. The developing eye provides a system to approach such a question. Biochemical and genetic studies have indicated that the planar polarity pathway downstream from Frizzled (Fz) and Dishevelled (Dsh) leads to the activation of a JNK-type MAPK module (Strutt et al. 1997; Boutros et al. 1998; Weber et al. 2000). During retinal morphogenesis, this pathway controls the mirror-symmetric arrangement of ommatidial units relative to the dorso-ventral midline. Thus, in the developing eye the activity of JNK and ERK signal transduction can be monitored separately in vivo (by planar polarity and R-cell recruitment, respectively).

To determine whether D-Fos is involved in planar polarity signaling, the effect of D-Fos ${ }^{\text {bZIP }}$ expressed under the control of Gal4 drivers in the developing eye was examined. When D-Fos function was thus reduced, a striking combined phenocopy of defects in ERK and JNK signal transduction ensued. Sections of eyes of the hairy Gal4/UAS D-fos ${ }^{\text {bZIP }}$ or of the sev Gal4/UAS D-fos ${ }^{\text {bZIP }}$ genotypes display both a lack of photoreceptor cells, diagnostic of inadequate ERK signal transduction, and misoriented ommatidia, indicating defects in planar polarity signaling (Fig. 5A-D). This mutant phenotype makes it plausible that D-Fos, in addition to its role in photoreceptor cell recruitment downstream from ERK, acts as an effector of JNK signaling in planar polarity determination. To confirm this, we tested whether the frequency of misoriented ommatidia that result when the Fz-Dsh-JNK planar polarity pathway is deregulated by overexpression of $\mathrm{Fz}$ can be suppressed when D-Fos function is reduced. Indeed, the mutant planar polarity phenotype is reverted almost to wild type in a heterozygous kay ${ }^{2}$ background (Fig. 5E,F). Similarly, the phenotypic defects caused when JNK signaling is stimulated by expression of activated Hep (Weber et al. 2000) in the eye imaginal disc can be suppressed by coexpression of DFos ${ }^{\text {bZIP }}$ or in a heterozygous kay mutant background (L. Ciapponi and U. Weber, unpubl.). These data indicate that D-Fos is, as shown earlier for its partner molecule D-Jun (Boutros et al. 1998), a nuclear effector of planar polarity signal transduction in eye development. There- 


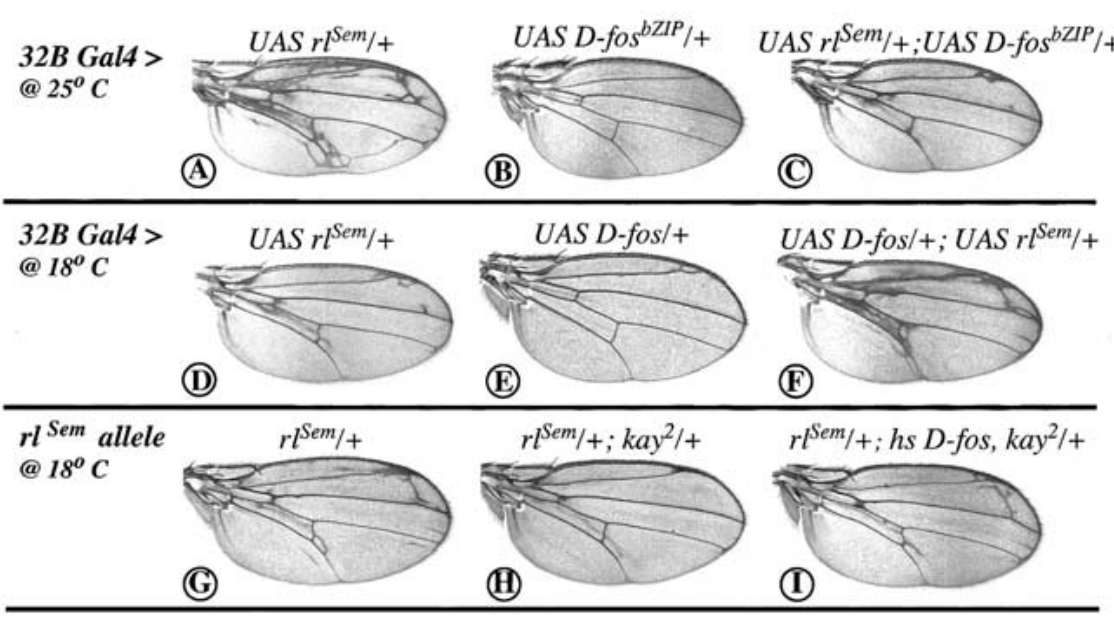
$(H)$ This can be suppressed almost completely by the kay ${ }^{2}$ allele. $(I)$ The suppression is overridden by the simultaneous expression of full-length D-Fos.

fore, D-Fos mediates both JNK and ERK responses in a defined group of cells of the developing retina.

\section{INK and ERK: phosphorylation site mapping on D-Fos}

The experiments discussed above indicate that D-Fos has multiple functions during Drosophila development downstream from (at least) two different signaling cascades. However, the mechanism by which JNK and ERK regulate D-Fos activity remains unclear. To investigate the potential regulation of D-Fos by protein phosphorylation, we performed in vitro kinase assays in which recombinant JNK/Bsk and ERK/Rl were used as kinases and different bacterially expressed versions of D-Fos
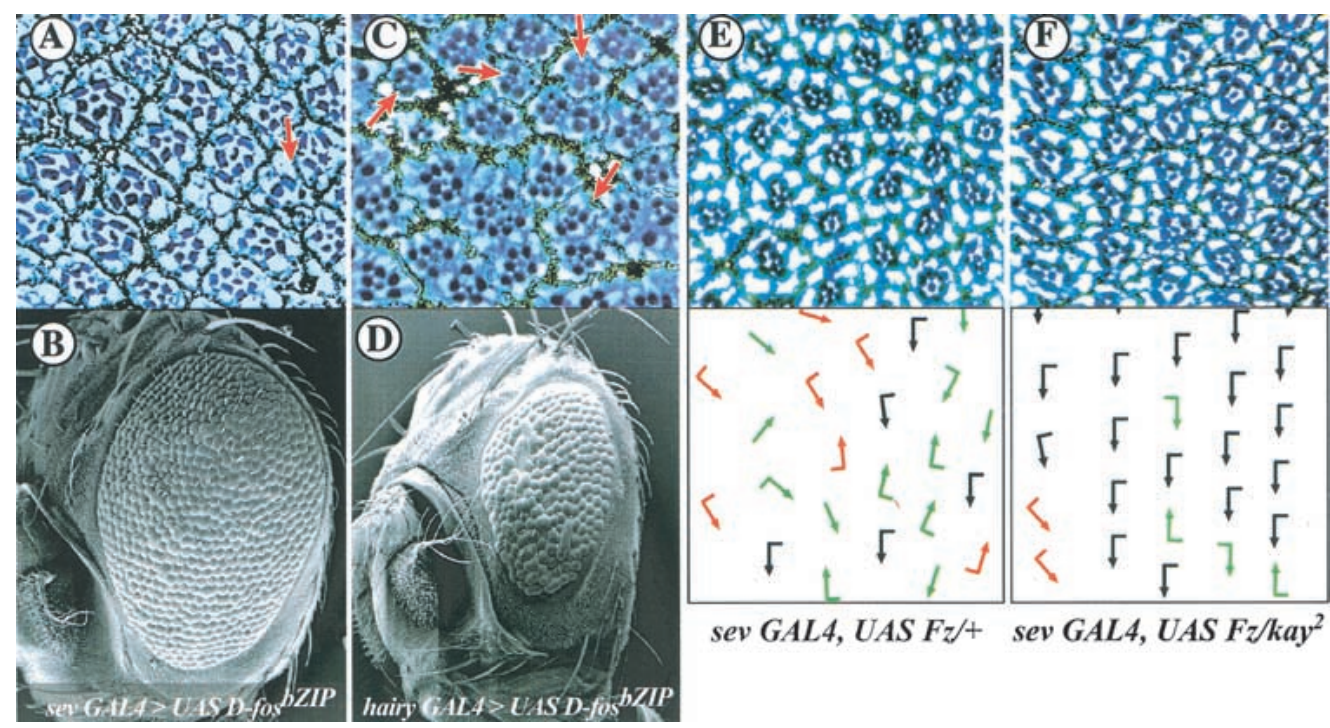

$\operatorname{sev} G A L 4, U A S F z /+\operatorname{sev} G A L 4, U A S F z / k a y^{2}$

Figure 5. D-Fos is an effector of both JNK and ERK pathways in the eye. Tangential eye sections of adult eyes of the genotypes sev $G A L 4>U A S f_{O S}{ }^{b Z I P}$ and hairy GAL4 $>U A S f_{O S}{ }^{b Z I P}(A$ and $C)$ and corresponding scanning electron micrographs $(B$ and $D)$. $(A)$ At the cellular level both photoreceptor recruitment (examples of ommatidia with less than seven photoreceptors are indicated by red arrows) and planar polarity (note the general misoriented organization of the ommatidia) are affected. $(B$ and $D)$ The expression of the mutant form of D-Fos in the eye causes external roughness. Note that the effect is stronger and accompanied by a reduced eye size when D-Fos ${ }^{\text {bZIP }}$ is under the control of the hairy GAL4 driver. This driver is active transiently during eye imaginal disc development, in all cells immediately anterior to the morphogenetic furrow, and in the presumptive R7 photoreceptor cells of the developing ommatidia (Brown et al. 1991), whereas the sev GAL4 driver is active mainly in the R7, 3, and 4 precursor cells. $(E$ and $F)$ Eye sections (top) and schematic representations (bottom), with arrows reflecting ommatidial planar polarity. (Black arrows) Correct ommatidial orientation; (red arrows) misrotated ommatidia with correct chirality; (green arrows) opposite chirality for the respective eye field; (arrows without flag) symmetrical nonchiral ommatidia. The disorganized appearance because of the overactivation of the planar polarity signaling cascade by sev GAL4, UAS Fz/+ (E) is suppressed dominantly by kay ${ }^{2}$ heterozygosity $(F)$. 
were used as substrates (Fig. 6C). In this in vitro setting, both Bsk and Rl could phosphorylate full-length D-Fos (Fig. 6A, lanes 2,12).

To get an initial indication which residues might serve as target sites for ERK or/and JNK, we compared the D-Fos amino acid sequence with that of mammalian Jun and Fos proteins. The sequence alignments identified several sequences in D-Fos with similarity to confirmed JNK or ERK phosphorylation sites in the mammalian molecules (data not shown). T89 and T93 of D-Fos correspond in their sequence context and relative location to established JNK phosphorylation sites in c-Jun (Papavassiliou et al. 1995). Alignment of the C-terminal parts of D-Fos and c-Fos showed a conserved residue (T584) that corresponds to a previously described MAPK phosphorylation site in c-Fos (Chen et al. 1993, 1996).
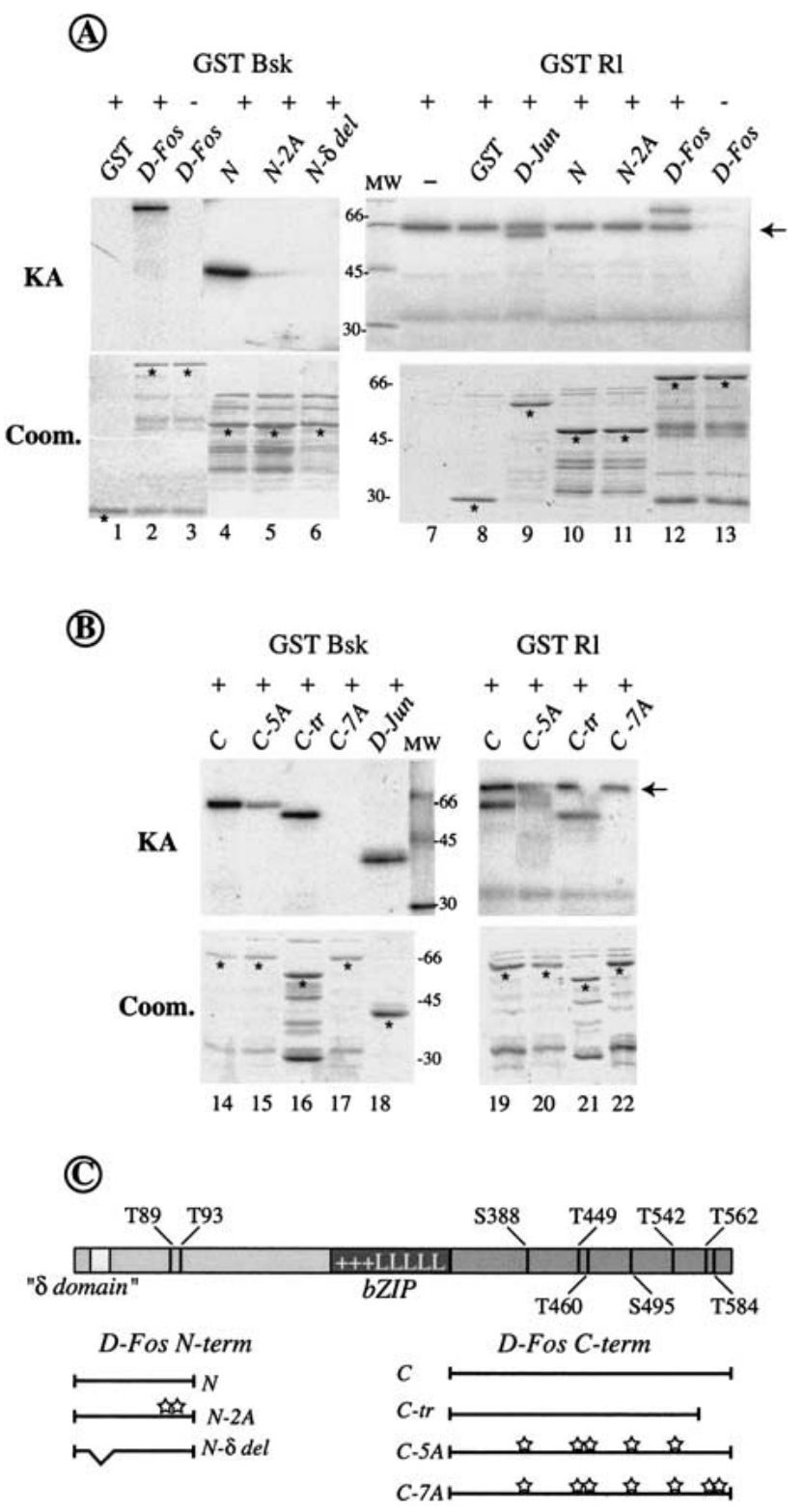

口 Ala substitution
Moreover, we identified several serine or threonine residues that might serve as target sites for the proline-directed MAPKs. We generated mutant derivatives of DFos in which one or more of these candidate phosphorylation sites was substituted by alanine (Fig. 6C). JNK/ Bsk, but not ERK/Rl, could efficiently phosphorylate a fragment spanning the $170 \mathrm{~N}$-terminal amino acids of D-Fos (Fig. 6A, lanes 4,10). When alanine substitutions were introduced in positions T89 and T93, the N-terminal D-Fos fragment was no longer an efficient substrate for JNK phosphorylation (Fig. 6A, lane 5).

Additional $\mathrm{N}$-terminal residues that conform to the S/TP consensus (T234, S235, T237, and T254) were phosphorylated by neither JNK nor ERK (data not shown). Similar to the 170-amino-acid N-terminal fragment tested above, a D-Fos fragment covering the N-terminal 285 amino acids was not a substrate for ERK (data not shown). This indicates that the $\mathrm{N}$-terminal part of D-Fos is a good substrate for JNK/Bsk but not for ERK/Rl, and that the residues T89 and T93 serve as the main N-terminal JNK target sites.

Interestingly, a small deletion that removes a sequence with remote similarity to the c-Jun $\delta$ domain (amino acids 28-56), but that does not span the phosphorylation sites T89 and T93 (Fig. 6C), completely abrogates phosphorylation of the $\mathrm{D}$-Fos $\mathrm{N}$-terminal fragment by JNK/Bsk (Fig. 6A, lane 6). It is possible that this deletion destroys a delta-domain-like JNK docking site present in D-Fos (Hibi et al. 1993).

Next, we analyzed the C-terminal part of D-Fos; it contains seven potential phosphorylation sites for

Figure 6. D-Fos contains distinct, but overlapping, sets of phosphorylation sites for JNK and ERK. Full-length D-Fos and different derivatives (described in $C$ ) were used as substrates for in vitro kinase assays (KA) with bacterially expressed Drosophila JNK (GST Bsk) or ERK (GST Rl). (A) Both JNK and ERK can phosphorylate full-length D-Fos in vitro (lanes 2,12). However, only Bsk can phosphorylate the D-Fos N-terminal fragment (lane 4), whereas ERK cannot (lane 10). Phosphorylation by JNK on the N-terminal part of D-Fos was lost completely when alanine substitutions in two putative phosphorylation sites or a deletion of a putative $\delta$ domain were introduced (lanes 5,6). (B) Both JNK and ERK can phosphorylate the C-terminal part of D-Fos (lanes 14,19), and this activity was blocked completely by seven alanine substitutions (lanes 17,22). Five alanine substitutions or a short C-terminal truncation are not enough to completely abolish the kinase activity on those substrates (lanes 15,16 and 20,21). Controls with GST (panel $A$, lanes 1,8), GST Jun (panel $A$, lane 9), or His Jun (panel $B$, lane 18) are shown. Note that the band at $66 \mathrm{kD}$ present in all lanes in the $\mathrm{ERK} / \mathrm{Rl}$ assays (arrow), is due to autophosphorylation of the kinase (panel $A$, cf. lanes 7 and 13 containing kinase but not substrate and vice versa, respectively). Comparable amounts of kinases and substrates (Bradford quantification) were used in all the experiments and confirmed by the Coomassie-stained gel below each kinase assay (Coom.). (Asterisk) Recombinant D-Fos proteins. (MW) The apparent molecular weight of size standards is indicated in $\mathrm{kD}$. (C) Schematic representation of D-Fos constructs. Alanine substitutions are indicated (stars). The positions of the phosphorylation sites and the bZIP region $(+++$ LLLLL) are shown. 
MAPKs (Fig. 6C). A fragment spanning the C-terminal 280 amino acids of D-Fos was, in contrast to the $\mathrm{N}$ terminus, phosphorylated efficiently by both Bsk and Rl (Fig. 6B, lanes 14,19). Alanine substitutions in all seven putative MAPKs target sites (Fig. 6C) caused complete loss of phosphorylation (Fig. 6B, lanes 17,22). However, mutating subsets of the seven putative phosphorylation sites, as in mutant C-5A or C-tr (Fig. 6C), did not result in a complete loss of phosphorylation by either $\mathrm{Rl}$ or Bsk (Fig. 6B, lanes 15,16,20,21), indicating the presence of multiple phosphorylation sites in the C-terminal part of D-Fos.

These results indicate that, at least in vitro, D-Fos is a direct substrate of both Drosophila JNK and ERK and that it contains overlapping, but distinct, sets of phosphorylation sites for the two kinases.

Differences in the phosphorylation state of D-Fos correlate with the choice between INK and ERK responses in vivo

After establishing which residues serve as substrates for JNK and/or ERK in vitro, it was important to determine the regulatory relevance of these sites in vivo. We generated transgenic fly strains expressing mutant forms of full-length D-Fos in which either the putative N-terminal, JNK-specific phosphorylation sites, or the C-terminally located ERK and JNK substrate sites were replaced by alanine (D-Fos ${ }^{\mathrm{N}-\mathrm{Ala}}$ and D-Fos ${ }^{\mathrm{C}-\mathrm{Ala}}$ in Fig. 7I, corresponding to N-2A and C-7A in Fig. 6C, respectively). In the D-Fos ${ }^{\text {pan Ala }}$ mutant, all the putative MAPK phosphorylation sites, both $\mathrm{N}$ - and C-terminal, were substituted by alanine (Fig. 7I). The transgenes in the lines tested gave rise to similar expression levels of the various D-Fos derivatives when tested by Western blot (Fig. 7J,K). The Western blot also shows that the phosphorylation site point mutants of D-Fos migrate at lower apparent molecular mass in the SDS gel when compared with the wild-type form of the protein. This is consistent with D-Fos being phosphorylated in vivo at the sites that were identified in the in vitro analysis.

When the nonphosphorylatable D-Fos ${ }^{\text {pan Ala }}$ was expressed from a UAS-driven transgene under the control of the epidermal driver 69B Gal4, it gave rise to a strong thoracic cleft at the dorsal midline, resembling kay or hep mutants, or phenotypes that result from D-Fos ${ }^{\text {bzIP }}$ expression (Fig. 7D). Evidently, D-Fos ${ }^{\text {pan Ala }}$ represents a dominant-negative mutant that can interfere with JNK-dependent thorax closure (Zeitlinger and Bohmann 1999).

Next, we investigated the relevance of subgroups of the D-Fos MAPK phosphorylation sites during thorax closure. Expression of D-Fos ${ }^{\mathrm{N}-\mathrm{Ala}}$ resulted in a distinctive thorax cleft, although not quite as pronounced as in the case of D-Fos ${ }^{\text {pan Ala }}$ (Fig. 7B). Importantly, this result shows that the N-terminal, JNK-specific phosphorylation sites in D-Fos are required for a well-defined JNKdependent developmental mechanism. Expression of D-
Fos ${ }^{\mathrm{C}-\mathrm{Ala}}$, which lacks the C-terminal phosphorylation sites, or of D-Fos ${ }^{\mathrm{wt}}$ had no discernible effect (Fig. 7C and data not shown). These results indicate that either the C-terminal sites play only an ancillary role and are not essential for JNK signaling in the signal transduction pathway controlling thorax, or that this mutant does not compete well with endogenous D-Fos and therefore has no dominant-negative effect in this context.

Next, we examined whether expression of the different phosphorylation mutants of D-Fos might also interfere with ERK responses. Expression of D-Fos ${ }^{\text {pan Ala }}$ in the posterior compartment of wing imaginal disc len Gal4, see Fig. 1 for expression pattern) caused loss of wing vein material typical of mutants defective in DER to Rolled signaling (Fig. $7 \mathrm{H}$ ). Thus, consistent with the observation that D-Fos ${ }^{\text {pan Ala }}$ has lost all substrate sites for both Rolled and Bsk, it acts as a dominant-negative form that interferes with D-Fos function in both the ERK and the JNK pathways (Fig. 7D,H).

Interestingly, however, the D-Fos ${ }^{\mathrm{N}-\mathrm{Ala}}$ and D-Fos ${ }^{\mathrm{C}-\mathrm{Ala}}$ mutants influence the ERK and JNK response differently. D-Fos ${ }^{\mathrm{N}-\mathrm{Ala}}$, which interfered dominantly with JNK-mediated thorax closure, had no effect on ERK-dependent wing vein formation (Fig. 7F). This is consistent with these sites not being substrates for Rolled. The DFos ${ }^{\text {C-Ala }}$ mutant, however, which was neutral in thorax development, caused loss-of-vein phenotype (Fig. 7G). It is important to note that the identical Fos ${ }^{\mathrm{Ala}}$ transgene lines were used in the thorax and the wing experiments. This, taken together with the results of the immunoblot analysis (Fig. 7M), makes it highly unlikely that the kinase pathway-specific effects of the different transgenes would be caused by differences in expression levels.

To examine whether differential phosphorylation of D-Fos might also be used in the developing eye to distinguish between ERK and JNK signaling, we analyzed the effect of the D-Fos ${ }^{\mathrm{Ala}}$ mutants on ERK-dependent photoreceptor cell recruitment and JNK-mediated ommatidial rotation. Different D-Fos ${ }^{\text {Ala }}$ mutants were expressed along with $\operatorname{sev}^{\mathrm{S} 11}$ in the eye imaginal disc under the control of the sevenless enhancer. As in the case of wing vein formation, D-Fos ${ }^{\mathrm{N}-\mathrm{Ala}}$ did not alter the $\mathrm{sev}^{\mathrm{S11}}$ phenotype, whereas the expression of D-Fos ${ }^{\mathrm{C}-\mathrm{Ala}}$ or of D-Fos ${ }^{\text {pan Ala }}$ caused a significant suppression of the extra R7-cell recruitment (Fig. 7J). Thus, ERK signaling, whether it is triggered by DER or by Sev, appears to require only the C-terminal phosphorylation sites of DFos.

Next, we tested whether the D-Fos ${ }^{\mathrm{Ala}}$ mutants could suppress the ommatidial misrotation phenotype that is elicited by overexpression of $\mathrm{Fz}$ and the ensuing activation of JNK. Coexpression of all Ala mutants and Fz under the control of sev Gal4 led to a significant suppression of the misrotation phenotype observed in flies expressing Fz alone (Fig. 7K). Wild-type D-Fos did not have this effect. These results indicate that the JNK-phosphorylation sites of D-Fos are required for the manifestation of the $\mathrm{Fz}$ gain-of-function phenotype. The Fz-JNK response in the eye is also affected by mutation of the 

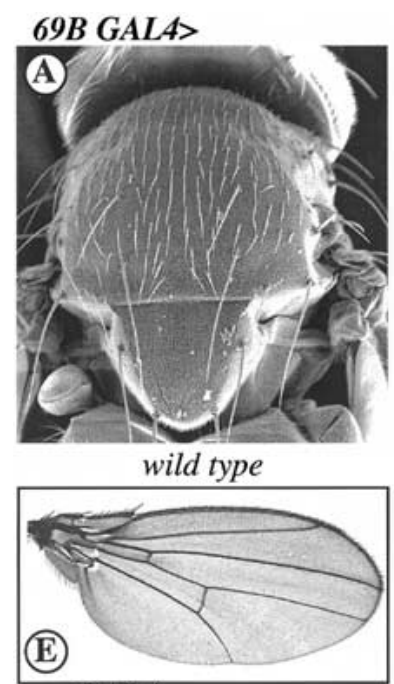

en GALA>

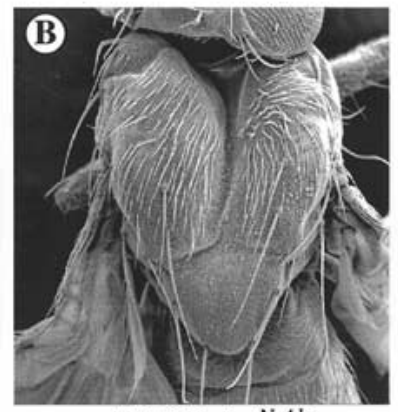

UAS D-fos ${ }^{N-A l a}$

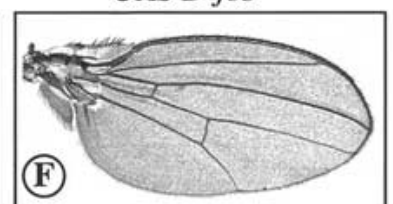

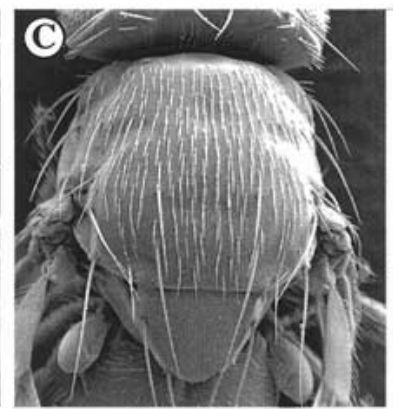

UAS D-fos ${ }^{C-A l a}$

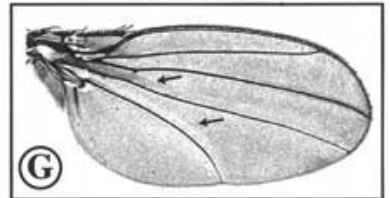

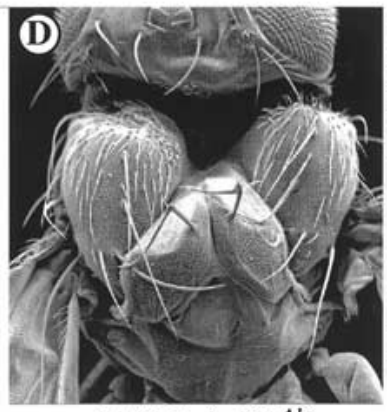

UAS D-fos pan Ala $^{\text {A }}$

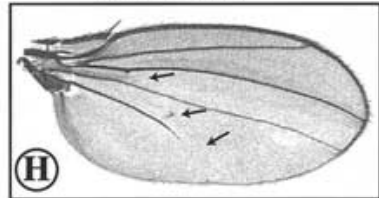

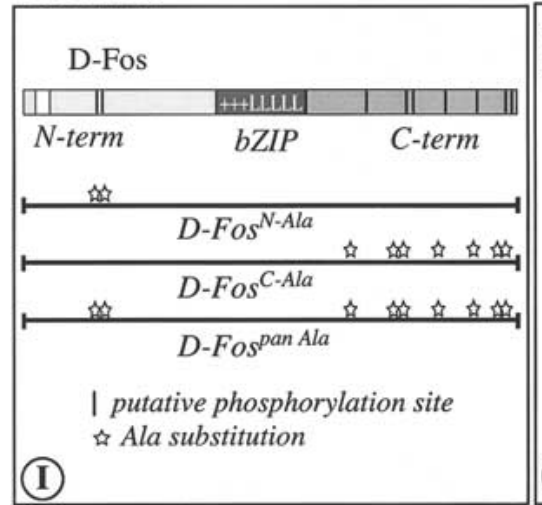
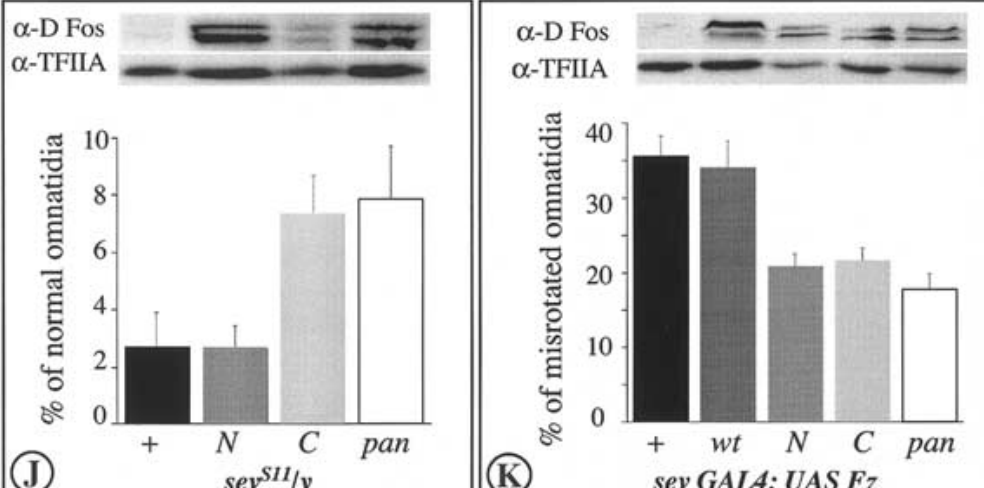

Figure 7. D-Fos MAPK phosphorylation sites are required differentially for in vivo functions. Scanning electron micrographs of adult thoraces $(A-D)$ and light micrographs of adult wings $(E-H)$. $(A)$ Wild-type thorax. $(B)$ expression of D-Fos ${ }^{\text {N-Ala }}$ (D-Fos, bearing alanine substitutions in the N-terminal JNK specific phosphorylation sites, see $I),(C)$ D-Fos ${ }^{\mathrm{C}-\mathrm{Ala}}$ (D-Fos, bearing alanine substitutions in the C-terminal MAPKs phosphorylation sites), or $(D)$ D-Fos ${ }^{\text {pan Ala }}$ (D-Fos, bearing alanine substitutions in all the MAPKs phosphorylation sites) under the control of $69 \mathrm{~B} \mathrm{GAL4}$ driver (at $25^{\circ} \mathrm{C}$ ). D-Fos ${ }^{\mathrm{N}-\mathrm{Ala}}$ and D-Fos ${ }^{\mathrm{pan}} \mathrm{Ala}$ cause a thorax phenotype reminiscent of a JNK defect. $(E)$ Wild-type wing. $(F)$ Expression of D-Fos ${ }^{\mathrm{N}-\mathrm{Ala}},(G)$ D-Fos ${ }^{\mathrm{C}-\mathrm{Ala}}$, or $(H)$ D-Fos ${ }^{\text {pan }}$ Ala under the control of en GAL4 driver $\left(\right.$ at $18^{\circ} \mathrm{C}$, with two copies). Only the expression of D-Fos containing alanine substitutions at the $\mathrm{C}$ terminus causes loss of vein material (arrows), a characteristic phenotype caused by defects in DER/ERK signaling. (I) Schematic representation of D-Fos ${ }^{\text {Ala }}$ mutants expressed in the transgenic flies. (Vertical bars) Putative phosphorylation sites; (stars) alanine substitutions. (J) Graphic representation of suppression of extra photoreceptor recruitment in gain-of-function sevenless mutants, sev ${ }^{\text {s11 }}$, by the different hs-D-Fos ${ }^{\text {Ala }}$ versions (bottom), and Western blot for the expression level (in wild-type background) of the various transgenes in the lines tested (top). The expression of $D$-fos bearing ERK phosphorylation sites mutated to alanine (D-Fos ${ }^{\mathrm{C}-\mathrm{Ala}}$ and D-Fos ${ }^{\mathrm{pan}}{ }^{\mathrm{Ala}} ; \mathrm{C}$ and pan in the graph) suppresses the sev $^{\mathrm{S11}}$ phenotype. However, alanine substitutions in JNK-specific phosphorylation sites $\left(\mathrm{D}\right.$-Fos $\left.{ }^{\mathrm{N}-\mathrm{Ala}} ; \mathrm{N}\right)$ do not affect the extra photoreceptor recruitment caused by activation of the RTK pathways. Larvae were heat shock treated at $37^{\circ} \mathrm{C}$ during the third instar larval stage for $30 \mathrm{~min}$. This caused the reduction of normal ommatidia in $s e v^{S 11} / y$ flies as compared with the same genotype analyzed in Fig. 2I. Percentage of normal ommatidia \pm S.D. is shown (for each genotype, at least three independent eyes and $>300$ ommatidia were analyzed, $P<0.0015)$. $(K)$ Graphic representation of suppression of misrotated ommatidia caused by the expression of frizzled under the control of sev GAL4 driver by the different UAS D-Fos ${ }^{\text {Ala }}$ versions (bottom), and a Western blot (top) monitoring the expression levels (using a ubiquitous driver, tub GAL4) of the various transgenes in the lines tested. The expression of any of the D-Fos ${ }^{\mathrm{Ala}}$ versions $\left(N, C\right.$, and pan), but not of D-Fos ${ }^{\mathrm{wt}}(w t)$, suppresses the sev GAL4; UAS Fz planar polarity phenotype. Percentage of misrotated ommatidia \pm S.D. is shown (for each genotype, at least three independent eyes and $>300$ ommatidia were analyzed, $P<0.0001$ ).

C-terminal sites that did not visibly disturb the thorax closure response. This might be explained by the higher sensitivity of the ommatidial rotation paradigm or a higher relative expression of the Fos ${ }^{\mathrm{Ala}}$ mutant transgene in the photoreceptor cells.

The dominant phenotypic effects of D-Fos ${ }^{\mathrm{Ala}}$ expression support our interpretation that Fos is regulated by protein phosphorylation to mediate developmental decisions and indicates that the residues identified by mutagenesis and in vitro kinase assay are required for in vivo function. Moreover, our findings indicate clearly that the function of D-Fos as a mediator of JNK/Bsk and $\mathrm{ERK} / \mathrm{Rl}$ cascades is in both cases that of a direct kinase substrate. 


\section{Discussion}

We have investigated several aspects of D-Fos function in signal transduction and development. Our experiments revealed that D-Fos is involved in a variety of developmental decisions controlled by ERK- and JNKtype MAP kinases. It will require further experiments to decide whether the findings on D-Fos can be extended to mammalian Fos proteins. As pointed out above, whereas the some of the phosphorylation sites described in this study appear to be conserved between D-Fos and c-Fos, for others this is less clear. Furthermore, a regulation of vertebrate Fos proteins by JNK-type MAP kinases has, so far, neither been shown conclusively nor refuted. One study has provided preliminary negative evidence arguing against a phosphorylation of c-Fos by JNK (Deng and Karin 1994). Similarly, it is not clear whether the regulation of Fos protein levels by changes in the activity of the coding gene or by signal-dependent alterations of Fos half-life, as they have been described for c-Fos, may be important in the control of D-Fos function.

The role of D-Fos as a mediator of wing vein formation and retinal planar polarity has so far not been described. Because of the cell lethality caused by the currently available $D$-fos null allele, we used the expression of a dominant-negative form of the protein for much of the functional analysis in the developing animal. Although this approach has proven very useful and experimentally versatile in previous studies of signal transduction and cell regulation, it is important to validate the obtained results and confirm that the observed effects are specific. Several lines of evidence indicate that this is the case. The effects of dominant-negative D-Fos were exacerbated in a $D$-fos heterozygous background. Furthermore, the dominant genetic interactions between mutant alleles of $D$-fos and other signal transduction components shown here, taken together with previous reports that illustrated the faithful reproduction of loss-of-function phenotypes in the embryo by dominant-negative D-Fos (Eresh et al. 1997; Zeitlinger et al. 1997), lend credence to the functional description of D-Fos during development presented here.

D-Fos cooperates with D-Jun presumably by forming a heterodimeric AP-1 complex. D-Jun is a substrate for Rl/ ERK and for Bsk/JNK (Peverali et al. 1996; Sluss et al. 1996). Therefore, it was not obvious that the function of D-Fos downstream from the MAP kinases was that of a direct substrate, as shown in this paper. It could have also been envisioned that D-Jun was the primary signal recipient, and D-Fos would play the role of a passive cofactor that aids DNA binding and transactivation. Why does Drosophila use both D-Fos and D-Jun as recipients of JNK or ERK signaling if ultimately they form a dimer anyway? First, it has been reported that Fos and Jun cooperate in gene activation, presumably by both supplying contact surfaces for components of the transcriptional machinery and, thus, contributing synergistically to a stable interaction (Abate et al. 1991). Even though the mechanistic consequences of D-Fos or D-Jun phosphorylation are not clear at present, it might be argued with the same logic that combining independent phosphoregulatable transactivation domains in a D-Fos-D-Jun heterodimer supports more effective gene activation in response to an upstream signal. Furthermore, indirect evidence has indicated that D-Fos might have D-Jun-independent functions, either as a homodimer that D-Fos, unlike its mammalian counterparts, can form, at least in vitro, or together with a third bZIP transcription factor (Perkins et al. 1990; RiesgoEscovar and Hafen 1997b; Szüts and Bienz 2000a). In such a scenario, D-Fos might need to be a MAP kinase target in its own right.

An important question raised by the finding that DFos can mediate signaling by both ERK and JNK is how the decision between these distinct cellular responses is made, that is, how the cell "knows" which program to execute when D-Fos becomes phosphorylated. Several mechanisms have been suggested to contribute to signal specificity in such situations, in which one protein mediates different cellular responses. One model proposes a combinatorial mechanism by which several factors with overlapping broad responsiveness have to cooperate to mediate a defined specific cellular behavior. However, the observation that D-Fos participates in ERK as well as JNK signal transduction in the same group of cells of the developing Drosophila eye, by regulating photoreceptor differentiation and ommatidial rotation, respectively, argues against cell type-specific cofactors that modulate the response to D-Fos phosphorylation. The distinct substrate sites in D-Fos phosphorylated by ERK and JNK raised a novel possibility to explain the signal-specific D-Fos response. We suggest that D-Fos exists in two different activated forms, depending on whether it was phosphorylated by ERK or JNK. These differentially phosphorylated forms might then selectively trigger either the ERK or the JNK response (Fig. 8). This idea is supported by in vivo experiments in which phosphorylation site-specific point mutants of D-Fos were expressed in the developing fly. A mutant that lacks all phosphorylation sites interferes dominantly with both ERK and JNK signaling in thorax closure, the wing, and the eye imaginal disc, supporting further the general relevance of D-Fos phosphorylation in developmental decisions. D-Fos mutants lacking subsets of phosphorylation sites, however, affected JNK and ERK signal responses differentially. An N-terminal cluster of JNK sites that is not phosphorylated by ERK was critical for the JNK response in vivo. A mutant lacking these sites interfered with thorax closure and planar polarity regulations, both bona fide JNK responses, but not with wing vein formation or photoreceptor differentiation, which are regulated by ERK. Conversely, a mutant that removes all ERK substrate sites dominantly suppresses processes normally controlled by this MAP kinase. These data indicate that signal-responsive transcription factors, such as D-Fos, may have different signal-specific functions. It is tempting to speculate that such a mechanism might be used by other signaling proteins that are receptive to different upstream signals.

A number of questions arise from this model of signal- 


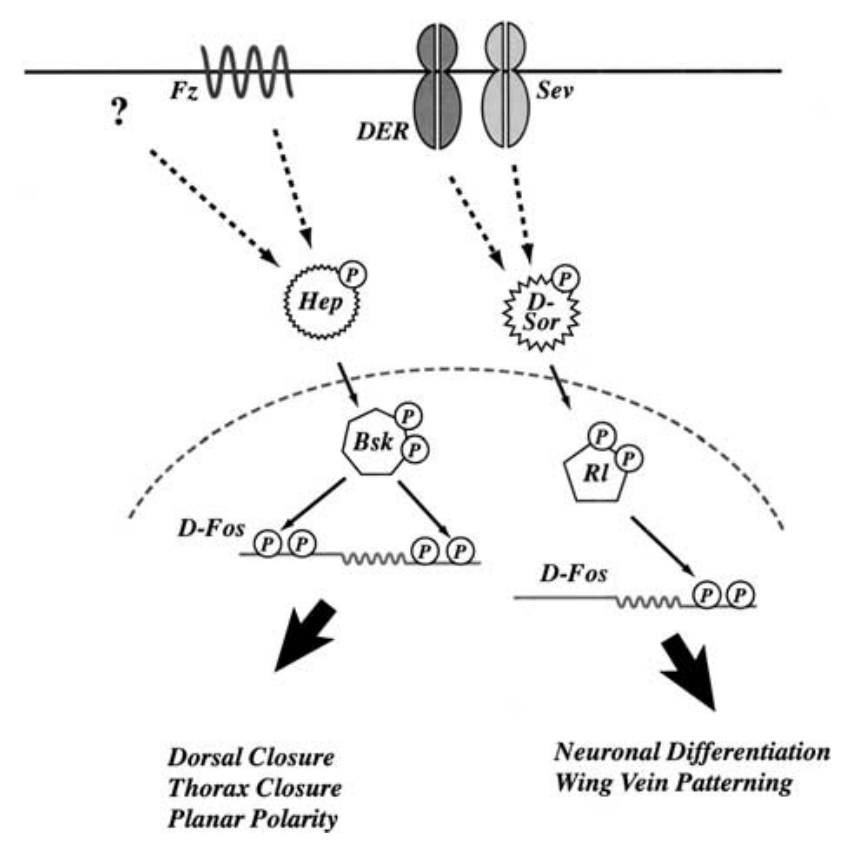

Figure 8. Model of phosphorylation specificity of D-Fos as mediator of the Drosophila JNK and ERK pathways. Schematic representation of the differences between JNK and ERK signal transduction via D-Fos. Distinct signal-dependent phosphoform of D-Fos may mediate the choice between JNK and ERK responses in vivo and initiate different biological programs.

specific transcription factor action: What mediates the different substrate specificities of JNK and ERK, and what are the molecular mechanisms responsible for the distinct readout of ERK- versus JNK-mediated D-Fos phosphorylation? The answer to the first question might be the presence of specific MAP kinase docking sites in the D-Fos polypeptide. It has been shown previously that the substrate specificity of MAP kinases is not mediated exclusively by the sequence surrounding the phosphoacceptor site, but also depends on protein regions that physically recruit MAPK to the vicinity of these sites (Minden and Karin 1997). In the case of D-Fos we have found evidence for such a docking element. Removal of a short amino acid sequence in the $\mathrm{N}$-terminal region that does not coincide with phosphorylation sites abrogates Basket phosphorylation. Hence, this element, which is distantly similar to the c-Jun $\delta$ domain, may mediate the JNK-specific phosphorylation in the N-terminal region of D-Fos.

The molecular basis for the differential readout of JNK and ERK signaling via D-Fos can only be speculated on at this time. It is conceivable that the differences in D-Fos phosphorylation affect the range of activated genes by the alternative pathways. This may be due to specific protein-protein interactions; for example, with other DNA-bound transcription factors required for the activation of JNK or ERK target genes. The interactions between different factors assembled on the enhancer of inducible genes (Maniatis et al. 1998) can be very intricate, and it is possible that they are sensitive to protein phos- phorylations in specific regions. More experiments involving, for example, genomic approaches will be required to identify the genes that are regulated by D-Fos in a signal- and cell context-specific manner.

\section{Materials and methods}

\section{Plasmid construction}

To generate mutant forms of D-Fos, the amino acids T88, T89, T93, T234, S235, T237, T254, S388, T449, T460, S495, T542, T562, and T584 were replaced in different combinations with alanine codons, by using the QuikChange Site-Directed Mutagenesis Kit (Stratagene), and sequenced. To generate the truncated D-Fos versions, standard PCR procedures were used. DFos N-terminal fragments used in kinase experiments spanned amino acids 1-170 or 1-285; the C-terminal fragment encompassed amino acids 315-595; the truncated form, amino acids $315-560$

\section{Bacterial expression vectors}

All truncated versions of D-Fos were cloned into the pGEX-3X expression vector (SmaI-EcoRI restriction sites) and sequenced. The rolled ${ }^{\text {Sem }}$ cDNA was cloned into the PGEX-2T expression vector (BamHI-EcoRI restriction sites) and subsequently sequenced. The GST-Bsk plasmid was a kind gift of H.K. Sluss (Sluss et al. 1996). GST D-jun, His-D-fos, and His-D-jun plasmids were kindly provided by F. Peverali (Peverali et al. 1996).

\section{P-element transformation vectors}

For the generation of transgenic flies expressing $D$-fos (Zeitlinger et al. 1997), D-fos ${ }^{N-A l a}, D-f o S^{C-A l a}, D-f o s^{p a n} A l a$, and $r l^{\mathrm{Sem}}$, cDNAs were cloned into the pUAST transformation vector (Brand and Perrimon 1993) or into the pKB267 P-element vector containing the sevenless enhancer and the heat shock promoter (Basler et al. 1991). UAS $D$-fos ${ }^{b Z I P}$ was obtained by cloning the basic region and the leucine zipper domain (amino acids 252-337) of $D$-fos into the pUAST transformation vector. D-fos ${ }^{\text {bZIP }}$ consists only of the bZIP domain; therefore, this mutant form can dimerize with endogenous Fos partner proteins such as D-Jun and bind to DNA, but it lacks the ability to stimulate transcription (Eresh et al. 1997).

\section{Transgenic and mutant Drosophila lines}

Germline transformation was performed by standard procedure (Spradling and Rubin 1982) using $w^{1118}$ as host strain and pUCHSpD2-3 (Mullins et al. 1990) as helper plasmid. The Gal4 drivers used in this study have been described previously (Brand and Perrimon 1993).

The following fly strains carrying sensitized or activated alleles of the receptor tyrosine kinase signaling pathway have been used for the suppression experiments: sev $^{\text {S11 }}$ (Basler et al. 1991); $r 1^{\text {Sem }}$ (Brunner et al. 1994); Elp $p^{B 1}$ (Baker and Rubin 1992).

\section{Bacterial expression of recombinant proteins}

The different GST D-Fos mutants, GST-R1 $1^{\text {Sem }}$ and GST-Bsk, were expressed in Escherichia coli BL21. Overnight LB cultures containing $100 \mu \mathrm{g} / \mathrm{mL}$ ampicillin were diluted 10-fold and grown for $1 \mathrm{~h}$ at $30^{\circ} \mathrm{C}$ (until $\mathrm{OD}_{600}=0.5-0.7$ ). Then the bacteria 
were induced with $1.5 \mathrm{mM}$ IPTG for $3 \mathrm{~h}$ at $30^{\circ} \mathrm{C}$. The bacteria were harvested and lysed for $30 \mathrm{~min}$ on ice in phosphate-buffered-saline (PBS) containing $100 \mu \mathrm{g} / \mathrm{mL}$ lysozyme, $1 \%$ Triton$\mathrm{X} 100$, and complete protease inhibitors (Sigma). After sonication (Branson sonifier 250) and centrifugation at 25,000g (Sorvall), the supernatant fraction was mixed with glutathioneSepharose beads. Bound proteins were eluted with reduced glutathione (GST purification modules; Pharmacia). Recombinant GST D-Jun, His-D-Jun, and His-D-Fos were purified as described previously (Peverali et al. 1996).

\section{In vitro protein kinase assay}

The kinase $(0.2 \mu \mathrm{g})$ and the different substrates $(1 \mu \mathrm{g})$ were incubated in $30 \mu \mathrm{L}$ (final volume) of kinase buffer (50 mM HEPES at $\mathrm{pH} 7.5,10 \mathrm{mM} \mathrm{MgCl} 2,1 \mathrm{mM}$ DTT, $25 \mathrm{mM} \beta$-glycerolphosphate, $0.2 \mathrm{mM}$ orthovanadate, $100 \mu \mathrm{M}$ ATP, $10 \mu \mathrm{Ci}$ of $\left[\gamma^{-}{ }^{32} \mathrm{P}\right]$ ATP). The phosphorylation reaction was terminated after 20 min at $30^{\circ} \mathrm{C}$ and loaded onto a $12 \%$ SDS-polyacrylamide gel. The SDS-polyacrylamide gel was developed first by Coomassie staining and subsequently by autoradiography. Under these conditions, GST-Bsk and GST-R1 ${ }^{\text {Sem }}$ (the constitutively active form of Rl) are sufficiently active on the substrates tested without phosphorylation by the respective MAPKK.

\section{Immunoblotting}

The transgenic lines bearing the different UAS D-Fos Ala or wild-type constructs (crossed with the tub Gal4 driver) or hs D-Fos Ala constructs were reared in cages on apple juice plates. Equal amounts of 0 to 14-h-old embryos of each genotype were collected and lysed in SDS sample buffer for $5 \mathrm{~min}$ at $98^{\circ} \mathrm{C}$ followed by centrifugation for $10 \mathrm{~min}$ at $15,000 \mathrm{~g}$. Aliquots of the supernatants were loaded onto $12 \%$ SDS-polyacrylamide gels. Western blots were performed according to Sambrook et al. (1990). Antibodies used were $\alpha$-D Fos (Zeitlinger et al. 1997) and $\alpha$-Drosophila TFIIA (Yokomori et al. 1993).

\section{Acknowledgments}

We thank Fiorenzo Peverali for several plasmids and recombinant proteins and Hayla K. Sluss for the GST-Bsk plasmid. We thank Ernst Hafen for $r l^{\mathrm{sem}}$ and $s e v^{S 11}$ fly strains. We are most grateful to Lutz Kockel and Julia Zeitlinger for their extraordinary support at the beginning of this work and for their continuous helpful discussion and advice. We would like to thank all the members of the Bohmann and Mlodzik laboratories for fruitful scientific interactions, in particular Ursula Weber, Uwe Gritzan, and Heinrich Jasper. We thank Silvia Sauer for technical assistance and Ann Marie Voie for embryo injections. Lutz Kockel, Julia Zeitlinger, Marco Milan, Catherine Ovitt, and all the members of the Bohmann laboratory made helpful comments on the manuscript. L.C. was supported by EMBO fellowship ATLF 334-1998.

The publication costs of this article were defrayed in part by payment of page charges. This article must therefore be hereby marked "advertisement" in accordance with 18 USC section 1734 solely to indicate this fact.

\section{References}

Abate, C., Luk, D., and Curran, T. 1991. Transcriptional regulation by Fos and Jun in vitro: Interaction among multiple activator and regulatory domains. Mol. Cell. Biol. 11: 36243632 .
Agnes, F., Suzanne, M., and Noselli, S. 1999. The Drosophila JNK pathway controls the morphogenesis of imaginal discs during metamorphosis. Development 126: 5453-5462.

Baker, N.E. and Rubin, G.M. 1989. Effect on eye development of dominant mutations in Drosophila homologue of the EGF receptor. Nature 340: 150-153.

- 1992. Ellipse mutations in the Drosophila homologue of the EGF receptor affect pattern formation, cell division, and cell death in eye imaginal discs. Dev. Biol. 150: 381-396.

Basler, K. and Hafen, E. 1988. Control of photoreceptor cell fate by the sevenless protein requires a functional tyrosine kinase domain. Cell 54: 299-311.

Basler, K., Christen, B., and Hafen, E. 1991. Ligand-independent activation of the sevenless receptor tyrosine kinase changes the fate of cells in the developing Drosophila eye. Cell 64: 1069-1081.

Biggs 3rd, W.H., Zavitz, K.H., Dickson, B., van der Straten, A., Brunner, D., Hafen, E., and Zipursky, S.L. 1994. The Drosophila rolled locus encodes a MAP kinase required in the sevenless signal transduction pathway. EMBO J. 13: 16281635.

Bohmann, D., Ellis, M.C., Staszewski, L.M., and Mlodzik, M. 1994. Drosophila Jun mediates Ras-dependent photoreceptor determination. Cell 78: 973-986.

Boutros, M., Paricio, N., Strutt, D.I., and Mlodzik, M. 1998. Dishevelled activates JNK and discriminates between JNK pathways in planar polarity and wingless signaling. Cell 94: $109-118$.

Brand, A.H. and Perrimon, N. 1993. Targeted gene expression as a means of altering cell fates and generating dominant phenotypes. Development 118: 401-415.

Brown, N.L., Sattler, C.A., Markey, D.R., and Carroll, S.B. 1991. hairy gene function in the Drosophila eye: Normal expression is dispensable but ectopic expression alters cell fates. Development 113: 1245-1256

Brunner, D., Oellers, N., Szabad, J., Biggs 3rd, W.H., Zipursky, S.L., and Hafen, E. 1994. A gain-of-function mutation in Drosophila MAP kinase activates multiple receptor tyrosine kinase signaling pathways. Cell 76: 875-588.

Chen, R.H., Abate, C., and Blenis, J. 1993. Phosphorylation of the c-Fos transrepression domain by mitogen-activated protein kinase and 90-kDa ribosomal S6 kinase. Proc. Natl. Acad. Sci. 90: 10952-10956.

Chen, R.H., Juo, P.C., Curran, T., and Blenis, J. 1996. Phosphorylation of c-Fos at the C-terminus enhances its transforming activity. Oncogene 12: 1493-1502.

Clifford, R.J. and T. Schüpbach, T. 1989. Coordinately and differentially mutable activities of torpedo, the Drosophila melanogaster homolog of the vertebrate EGF receptor gene. Genetics 123: 771-787.

De Cesare, D., Fimia, G.M., and Sassone-Corsi, P. 1999. Signaling routes to CREM and CREB: Plasticity in transcriptional activation. Trends Biochem. Sci. 24: 281-285.

Deng, T. and Karin, M. 1994. c-Fos transcriptional activity stimulated by H-Ras-activated protein kinase distinct from JNK and ERK. Nature 371: 171-175.

Dérijard, B., Hibi, M., Wu, I., Barrett, T., Su, B., Deng, T., Karin, M., and Davis, R. 1994. JNK1: A protein kinase stimulated by UV-light and Ha-Ras that binds and phosphorylates the c-Jun activation domain. Cell 76: 1025-1037.

Diaz-Benjumea, F.J. and Hafen, E. 1994. The sevenless signalling cassette mediates Drosophila EGF receptor function during epidermal development. Development 120: 569578.

Eresh, S., Riese, J., Jackson, D.B., Bohmann, D., and Bienz, M. 1997. A CREB-binding site as a target for decapentaplegic 
signalling during Drosophila endoderm induction. EMBO J. 16: $2014-2022$.

Fortini, M.E., Simon, M.A., and Rubin, G.M. 1992. Signalling by the sevenless protein tyrosine kinase is mimicked by Ras1 activation [see comments]. Nature 355: 559-561.

Freeman, M. 1996. Reiterative use of the EGF receptor triggers differentiation of all cell types in the Drosophila eye. Cell 87: 651-660.

1998. Complexity of EGF receptor signalling revealed in Drosophila. Curr. Opin. Genet. Dev. 8: 407-411.

Gabay, L., Seger, R., and Shilo, B.Z. 1997. MAP kinase in situ activation atlas during Drosophila embryogenesis. Development 124: 3535-3541.

Garcia-Bellido, A., Cortes, F., and Milan, M. 1994. Cell interactions in the control of size in Drosophila wings. Proc. Natl. Acad. Sci. 91: 10222-10226.

Hibi, M., Lin, A., Smeal, T., Minden, A., and Karin, M. 1993. Identification of an oncoprotein- and UV-responsive protein kinase that binds and potentiates the c-Jun activation domain. Genes \& Dev. 7: 2135-2148.

Hou, X.S., Goldstein, E.S., and Perrimon, N. 1997. Drosophila Jun relays the Jun amino-terminal kinase signal transduction pathway to the Decapentaplegic signal transduction pathway in regulating epithelial cell sheet movement. Genes \& Dev. 11: 1728-1737.

Knust, E. 1996. Drosophila morphogenesis: Follow-my-leader in epithelia. Curr. Biol. 6: 379-381.

Kockel, L., Zeitlinger, J., Staszewski, L.M., Mlodzik, M., and Bohmann, D. 1997. Jun in Drosophila development: Redundant and nonredundant functions and regulation by two MAPK signal transduction pathways. Genes \& Dev. 11: 1748-1758.

Kockel, L., Homsy, J.G., and Bohmann, D. 2001. Drosophila AP-1: Lesson from an invertebrate. Oncogene (in press).

Leppä, S. and Bohmann, D. 1999. Diverse functions of JNK signaling and c-Jun in stress response and apoptosis. Oncogene 18: 6158-6162.

Leppä, S., Saffrich, R., Ansorge, W., and Bohmann, D. 1998. Differential regulation of c-Jun by ERK and JNK during PC12 cell differentiation. EMBO J. 17: 4404-4413.

Lim, Y.M., Tsuda, L., Inoue, Y.H., Irie, K., Adachi-Yamada, T., Hata, M., Nishi, Y., Matsumoto, K., and Nishida, Y. 1997. Dominant mutations of Drosophila MAP kinase kinase and their activities in Drosophila and yeast MAP kinase cascades. Genetics 146: 263-273.

Maniatis, T., Falvo, J.V., Kim, T.H., Kim, T.K., Lin, C.H., Parekh, B.S., and Wathelet, M.G. 1998. Structure and function of the interferon- $\beta$ enhanceosome. Cold Spring Harb. Symp. Quant. Biol. 63: 609-620.

Martin-Blanco, E., Pastor-Pareja, J.C., and Garcia-Bellido, A. 2000. From the cover: JNK and decapentaplegic signaling control adhesiveness and cytoskeleton dynamics during thorax closure in Drosophila. Proc. Natl. Acad. Sci. 97: 78887893.

Minden, A. and Karin, M. 1997. Regulation and function of the JNK subgroup of MAP kinases. Biochim. Biophys. Acta. 1333: F85-F104.

Mullins, M.C., Rio, D.C., and Rubin, G.M. 1990. Cis-acting DNA sequence requirements for P-element transposition. Genes \& Dev. 3: 729-738.

Noselli, S. 1998. JNK signaling and morphogenesis in Drosophila. Trends Genet. 14: 33-38.

Papavassiliou, A.G., Treier, M., and Bohmann, D. 1995. Intramolecular signal transduction in c-Jun. EMBO J. 14: 20142019.
Perkins, K.K., Admon, A., Patel, N., and Tjian, R. 1990. The Drosophila fos-related AP-1 protein is a developmentally regulated transcription factor. Genes \& Dev. 4: 822-834.

Peverali, F.A., Isaksson, A., Papavassiliou, A.A., Plastina, P., Staszewski, L.M., Mlodzik, M., and Bohmann, D. 1996. Phosphorylation of Drosophila Jun by the MAP kinase rolled regulates photoreceptor differentiation. EMBO J. 15: 39433950.

Pulverer, B.J., Kyriakis, J.M., Aruch, J., Nikolakaki, E., and Woodgett, J.R. 1991. Phosphorylation of c-Jun mediated by MAP kinase. Nature 353: 670-674.

Riese, J., Tremml, G., and Bienz, M. 1997. D-Fos, a target gene of Decapentaplegic signaling required during Drosophila endoderm induction. Development 124: 3353-3361.

Riesgo-Escovar, J.R. and Hafen, E. 1997a. Common and distinct roles of DFos and DJun during Drosophila development. Science 278: 669-672.

- 1997b. Drosophila Jun kinase regulates expression of decapentaplegic via the ETS-domain protein Aop and the AP-1 transcription factor DJun during dorsal closure. Genes \& Dev. 11: 1717-1727.

Riesgo-Escovar, J.R., Jenni, M., Fritz, A., and Hafen, E. 1996. The Drosophila Jun-N-terminal kinase is required for cell morphogenesis but not for DJun-dependent cell fate specification in the eye. Genes \& Dev. 10: 2759-2768.

Sambrook, J., Fritsch, E.F., and Maniatis, T. 1990. Molecular cloning: A laboratory manual. Cold Spring Harbor Laboratory Press, Cold Spring Harbor, NY.

Schweitzer, R. and Shilo, B.Z. 1997. A thousand and one roles for the Drosophila EGF receptor. Trends Genet. 13: 191-196.

Simon, M.A. 1994. Signal transduction during the development of the Drosophila R7 photoreceptor. Dev. Biol. 166: 431442 .

2000. Receptor tyrosine kinases: Specific outcomes from general signals. Cell 103: 13-15.

Sluss, H.K. and Davis, R.J. 1997. Embryonic morphogenesis signaling pathway mediated by JNK targets the transcription factor JUN and the TGF- $\beta$ homologue decapentaplegic. $J$. Cell. Biochem. 67: 1-12.

Sluss, H.K., Han, Z., Barrett, T., Davis, R.J., and Ip, Y.T. 1996. A JNK signal transduction pathway that mediates morphogenesis and an immune response in Drosophila. Genes \& Dev. 10: $2745-2758$.

Spradling, A.C. and Rubin, G.M. 1982. Transposition of cloned P-elements into Drosophila germline chromosomes. Science 218: 341-347.

Stronach, B.E. and Perrimon, N. 1999. Stress signaling in Drosophila. Oncogene 18: 6172-6182.

Strutt, D.I., Weber, U., and Mlodzik, M. 1997. The role of RhoA in tissue polarity and Frizzled signalling. Nature 387: 292295.

Sturtevant, M.A., Roark, M., and Bier, E. 1993. The Drosophila rhomboid gene mediates the localized formation of wing veins and interacts genetically with components of the EGF-R signaling pathway. Genes \& Dev. 7: 961-973.

Szüts, D. and Bienz, M. 2000a. LexA chimeras reveal the function of Drosophila Fos as a context-dependent transcriptional activator. Proc. Nat1. Acad. Sci. 97: 5351-5356.

. 2000b. An autoregulatory function of Dfos during Drosophila endoderm induction. Mech. Dev. 98: 71-76.

Szüts, D., Eresh, S., and Bienz, M. 1998. Functional intertwining of Dpp and EGFR signaling during Drosophila endoderm induction. Genes \& Dev. 12: 2022-2035.

Treier, M., Bohmann, D., and Mlodzik, M. 1995. JUN cooperates with the ETS domain protein pointed to induce 
photoreceptor R7 fate in the Drosophila eye. Cell 83: 753760.

Treisman, R. 1996. Regulation of transcription by MAP kinase cascades. Curr. Opin. Cell Biol. 8: 205-215.

Wassarman, D.A., Therrien, M., and Rubin, G.M. 1995. The Ras signaling pathway in Drosophila. Curr. Opin. Genet. Dev. 5: 44-50.

Weber, U., Paricio, N., and Mlodzik, M. 2000. Jun mediates Frizzled-induced R3/R4 cell fate distinction and planar polarity determination in the Drosophila eye. Development 127: 3619-3629.

Wisdom, R. 1999. AP-1: One switch for many signals. Exp. Cell Res. 253: 180-185.

Yokomori, K., Admon, A., Goodrich, J.A., Chen, J.L., and Tjian, R. 1993. Drosophila TFIIA-L is processed into two subunits that are associated with the TBP/TAF complex. Genes \& Dev. 7: 2235-2245.

Zeitlinger, J. and Bohmann, D. 1999. Thorax closure in Drosophila: Involvement of Fos and the JNK pathway. Development 126: 3947-3956.

Zeitlinger, J., Kockel, L., Peverali, F.A., Jackson, D.B., Mlodzik, M., and Bohmann, D. 1997. Defective dorsal closure and loss of epidermal decapentaplegic expression in Drosophila fos mutants. EMBO J. 16: 7393-7401. 


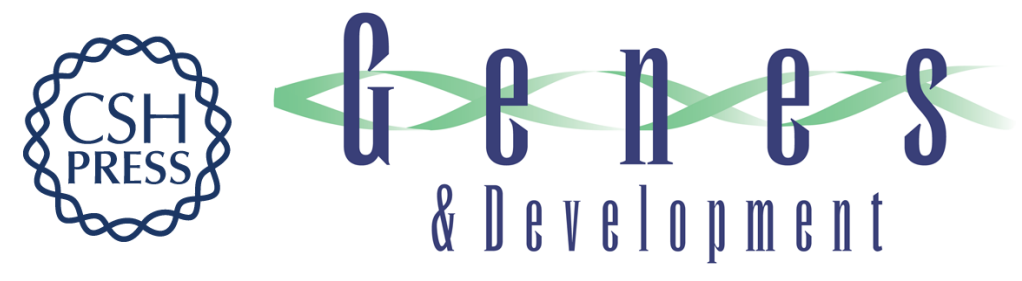

\section{Drosophila Fos mediates ERK and JNK signals via distinct phosphorylation sites}

Laura Ciapponi, David B. Jackson, Marek Mlodzik, et al.

Genes Dev. 2001, 15:

Access the most recent version at doi:10.1101/gad.886301

References

This article cites 65 articles, 31 of which can be accessed free at: http://genesdev.cshlp.org/content/15/12/1540.full.html\#ref-list-1

License

Email Alerting

Receive free email alerts when new articles cite this article - sign up in the box at the top Service right corner of the article or click here.

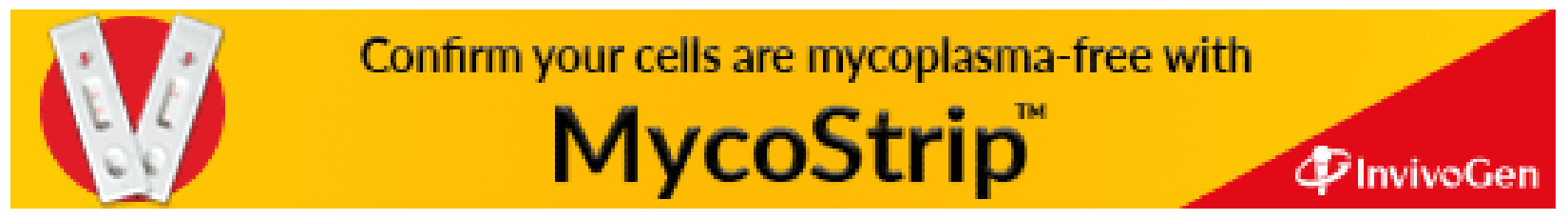

